\title{
A HaloTag Anchored Ruler for Week-Long Studies of Protein Dynamics
}

\section{SUPPORTING INFORMATION}

Ionel Popa ${ }^{* 1}$, Jaime Andrés Rivas-Pardo, Edward C. Eckels, Daniel Echelman, Carmen L. Badilla, Jessica Valle-Orero, Julio M. Fernández*

Department of Biological Sciences, Columbia University, 1212 Amsterdam Avenue, New York, New York 10027, United States.

E-mail: popa@uwm.edu,jfernandez@columbia.edu

${ }^{1}$ Present address: Department of Physics, University of Wisconsin-Milwaukee, 3135 North Maryland Ave., Milwaukee, WI 53211 


\section{SUPPLEMENTARY MATERIALS AND METHODS}

\section{Image processing}

Magnetic tweezers depend on image processing to determine the z-position of the two beads: a paramagnetic bead to which the protein is tethered, and a non-magnetic fixed reference bead which is used to subtract out instrumental vibration and focal drift. Several approaches based on highly parallelized computing using the computer's graphic processing units have been developed ${ }^{1}$. Here we chose a simpler approach, which uses solely the computer's central processing unit (CPU) to calculate in real-time the position of the beads. To achieve satisfactory results, we have both overclocked the CPU (i7 Intel CPU, overclocked to $4.6 \mathrm{GHz}$ ) and optimized the image processing algorithms.

The main steps of our program are: acquisition of a frame by a camera and its transfer to the computer, obtaining the region of interest (ROI) around the beads of interest (of $128 \mathrm{x} 128 \mathrm{px}^{2}$ ), computing Fourier transforms (FFT) from the two ROIs, calculating the radial profile and the correlation with the stack library and fitting the correlation profile to determine the position of each bead (Figure S1).

The slowest two steps in our approach are the computation of the FFT and its radial profile. To improve the calculation of the radial profile, we utilize a pixel-addressing algorithm (FKA algorithm). Figure S1 offers a simplified illustration of the pixel-addressing algorithm in a generated 128x128 pixel image. Its corresponding FFT is a matrix of $65 \times 128$ pixels (Figure S1B). We obtain the radial profile address function $r_{0}$ of the pixel coordinates:

$$
r_{0}=p \cdot\left\|\sqrt{\left(W_{x}-x_{c}\right)^{2}+\left(W_{y}-y_{c}\right)^{2}}\right\|, \quad \text { Equation S1 }
$$

where $p$ is a digital zoom factor ( $p=4$ in Figure S1), $W_{x}$ and $W_{y}$ are matrixes having the same number of elements as the FFT matrix, with their rows and columns respectively increasing in increments of one, and $x_{c}$ and $y_{c}$ are the expected centers of the FFT. Using the address matrix we can compute the value of each point in the radial profile by simply averaging the pixels within a given address number.

FKA algorithm: To reduce the computation time during image processing, we wrote a specified pixel-addressing algorithm (FKA algorithm) to construct the radial profile from an FFT. Given that each pixel always contributes to a unique radial position, we can easily calculate all the values in the radial vector by averaging the intensities (I0) of the contributing pixels. There are exactly 8320 pixels in a 128 x 65 FFT (Figure S1B). Below, we compute the radial vector (x4) contributions to positions 48, 94 and 140 (black pixels in Figure S1B and the corresponding circles in Figure S1C):

Profile[48]=(I0[3380]+I0[3381]+I0[3450]+I0[3583]+IO[3649]+IO[3846]+I0[4107]+ $\mathrm{IO}[4172]+\mathrm{IO}[4237]+\mathrm{I} 0[4496]+\mathrm{I} 0[4689]+\mathrm{IO}[4753]+\mathrm{IO}[4880]+\mathrm{IO}[4940]+\mathrm{IO}[4941]) / 15$ Profile[94] $=(\mathrm{I0}[2670]+\mathrm{I} 0[2738]+\mathrm{I} 0[2939]+\mathrm{I} 0[3005]+\mathrm{IO}[3203]+\mathrm{IO}[3269]+\mathrm{I} 0[3662]+$ $\mathrm{I} 0[3858]+\mathrm{I} 0[4508]+\mathrm{I} 0[4702]+\mathrm{I} 0[5089]+\mathrm{I} 0[5153]+\mathrm{I} 0[5345]+\mathrm{I} 0[5409]+\mathrm{I} 0[5598]+\mathrm{I} 0[$ $5660]) / 16$ 
Profile[140]=(IO[1885]+I0[1886]+I0[1887]+IO[1958]+I0[2027]+IO[2094]+IO[2161] $+\mathrm{IO}[2228]+\mathrm{IO}[2361]+\mathrm{IO}[2823]+\mathrm{I} 0[3020]+\mathrm{I} 0[3151]+\mathrm{I} 0[3282]+\mathrm{I} 0[3413]+\mathrm{I} 0[3674]+\mathrm{I} 0$ [4065]+I0[4130]+IO[4195]+IO[4260]+IO[4325]+IO[4714]+I0[4973]+IO[5102]+IO[52 $31]+\mathrm{I} 0[5360]+\mathrm{I} 0[5553]+\mathrm{I} 0[6001]+\mathrm{I} 0[6128]+\mathrm{I} 0[6191]+\mathrm{IO}[6254]+\mathrm{IO}[6317]+\mathrm{IO}[6378]$ $+\mathrm{I}[6435]+\mathrm{I} 0[6436]+\mathrm{I} 0[6437]) / 35$

In our experiments, the change in the end-to-end length of the tethered protein is determined from the diffraction pattern around the bead, which is highly sensitive to the position of the bead in the Z-plane. We first select regions of interest (ROI) of 128x128 pixel around the addressed beads, and apply a Kaiser window to reduce artifacts from the boundaries of the image ${ }^{2}$ (Figure S1A). We then compute the FFT of the bead, which centers the signal at the origin, eliminating $x y$ fluctuations (Figure S1B). We then applied our pixel-addressing algorithm (FKA) to compute the radial profile of the FFT image (Figure S1C). For our experiments we obtain the radial profile using a digital zoom factor of $p=4$, which further increases the resolution. For beads with diameter of $\sim 3 \mu \mathrm{m}$ measured at magnifications of $100 \mathrm{x}$ we find that the range between 40 to 140 points in the profile gives the best results. This range ignores the low frequencies, which are dominated by the bead position, and the high frequency spectra, which are altered by the camera sampling and acquisition noise ${ }^{3}$. We chose a paramagnetic and reference bead from the field of view. To determine the z-position of both beads we correlate their radial profile against a stack library. Radial profile stack libraries are obtained at $20 \mathrm{~nm}$ increments by stepping the position of the objective on a piezoelectric actuator, and are recorded for each bead prior to the measurement (Figure S1D). During the measurement, the real-time position of the bead is computed from the Gaussian fit of the Pearson correlations between the current radial profile and the stack library (Figure S1E). This method resolves the z-position of a bead with nanometer resolution. 


\section{SUPPLEMENTARY FIGURES}

\section{FIGURE S1}
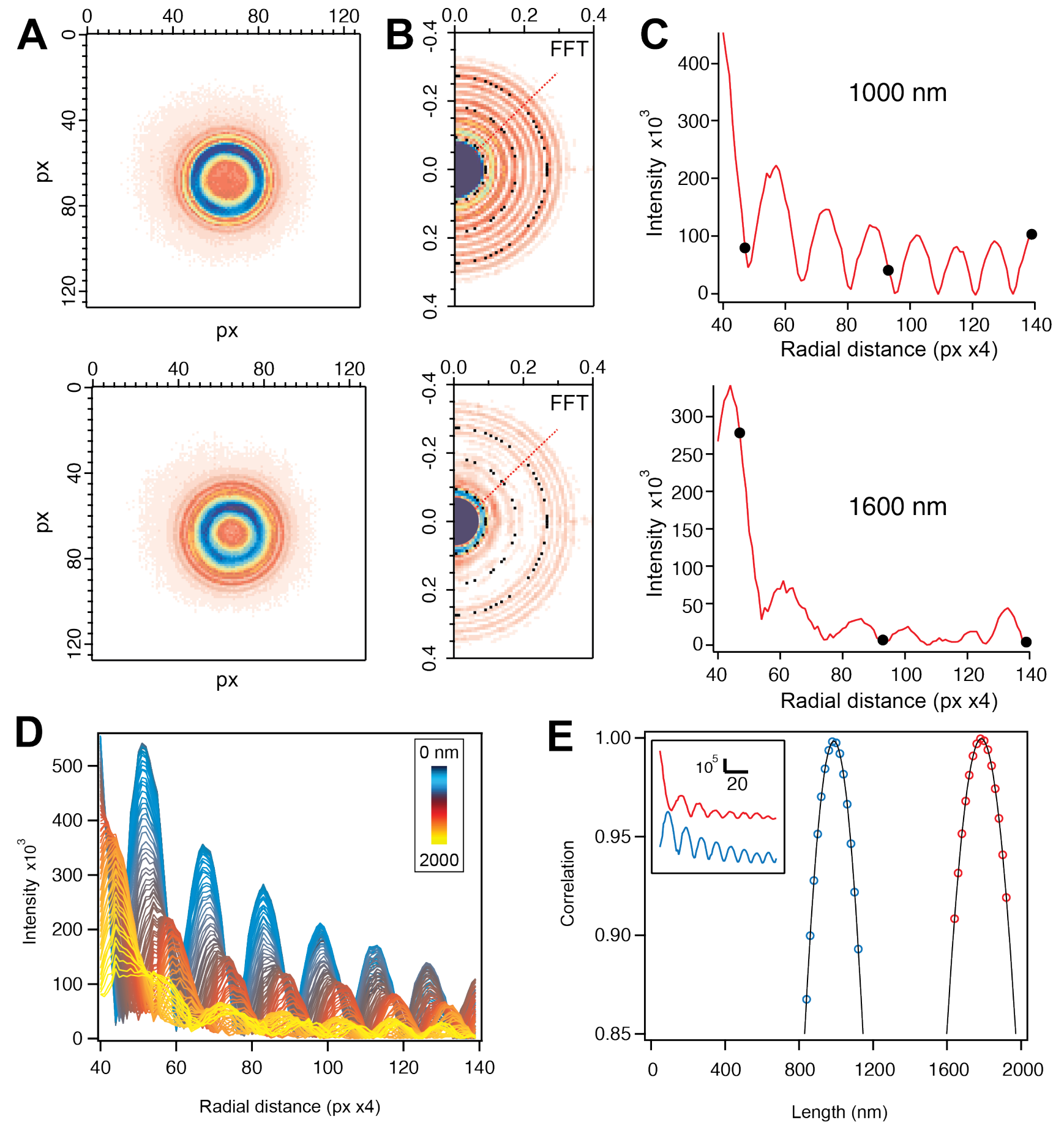

Figure S1. Measuring the z-position of beads, using image processing. A) $128 \times 128$ pixel ROI of a $2.8 \mu \mathrm{m}$ bead at focal position $1000 \mathrm{~nm}$ (top) and $1600 \mathrm{~nm}$ (bottom). B) FFT of the images in (A). The red line marks a radial vector. The black dots mark the pixels that contribute to three different radii: 48, 94,140 (pixels x4). C) Radial profiles resulting from the FFT's shown in (B) and computed using the FKA algorithm. The black dots identify radial vector positions 48 , 
94, 140 (see B). D) Stack of radial vectors obtained by moving the objective focal plane (z position) through the bead shown in (A) over $2 \mu \mathrm{m}$, in steps of $20 \mathrm{~nm}$. E) Correlation profiles between measured radial vectors (inset) and the stack at two different $\mathrm{z}$ positions. The correlations are fit with a Gaussian distribution (solid lines), where the mean reports the location of the bead in the z-axis. 


\section{FIGURE S2}
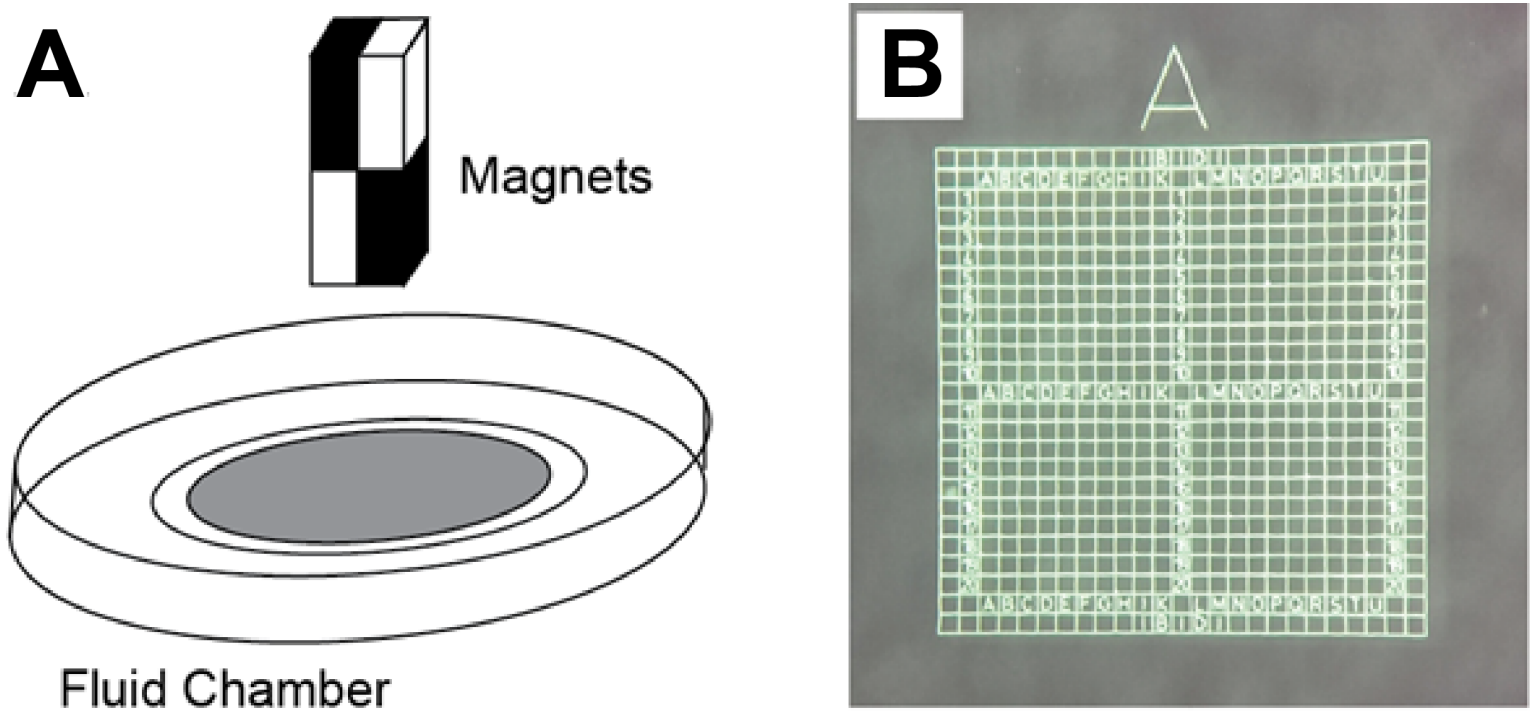

\section{Fluid Chamber}
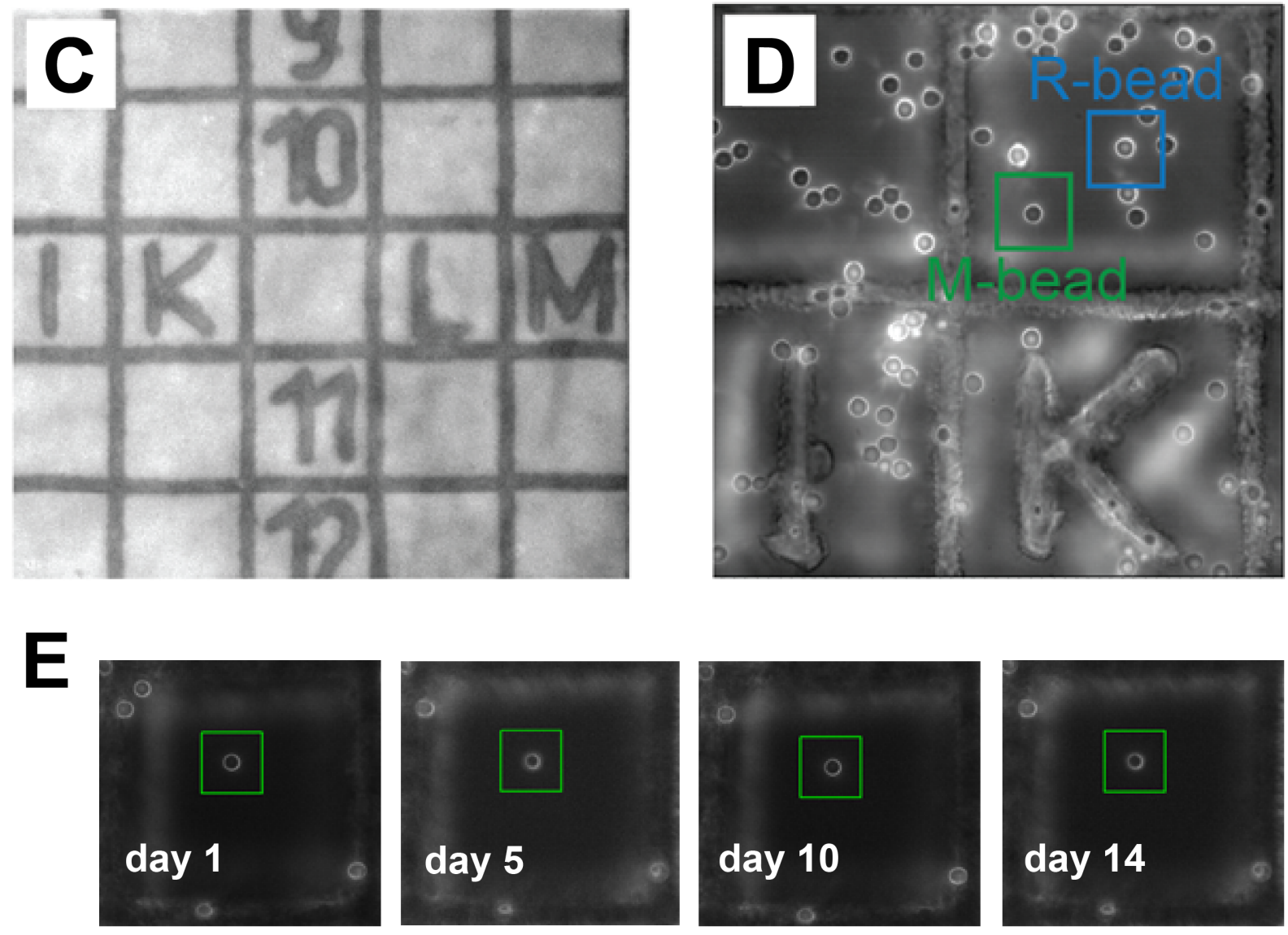

Figure S2. Chamber design for long-term single molecule measurements. A) Schematic of Ibidi $\mu$ Dish fluid chamber positioned beneath a set of permanent magnets. B) Magnification of the 
$25 \times 25$ grid array at the center of the fluid chamber. Each cell is $50 \mu^{2}$. C) Cells in the grid array are addressable by alphanumeric coordinates. D) Screenshot of the grid showing the reference (R, blue square) and paramagnetic (M, green square) beads.E) A) Images of the paramagnetic bead (green square) probed over a period of 14 days (Figure 5). The bead was readily addressable each day in grid position G17 at $x-y$ coordinates of $12.99 \mathrm{~mm}, 9.60 \mathrm{~mm}$. 


\section{FIGURE S3}

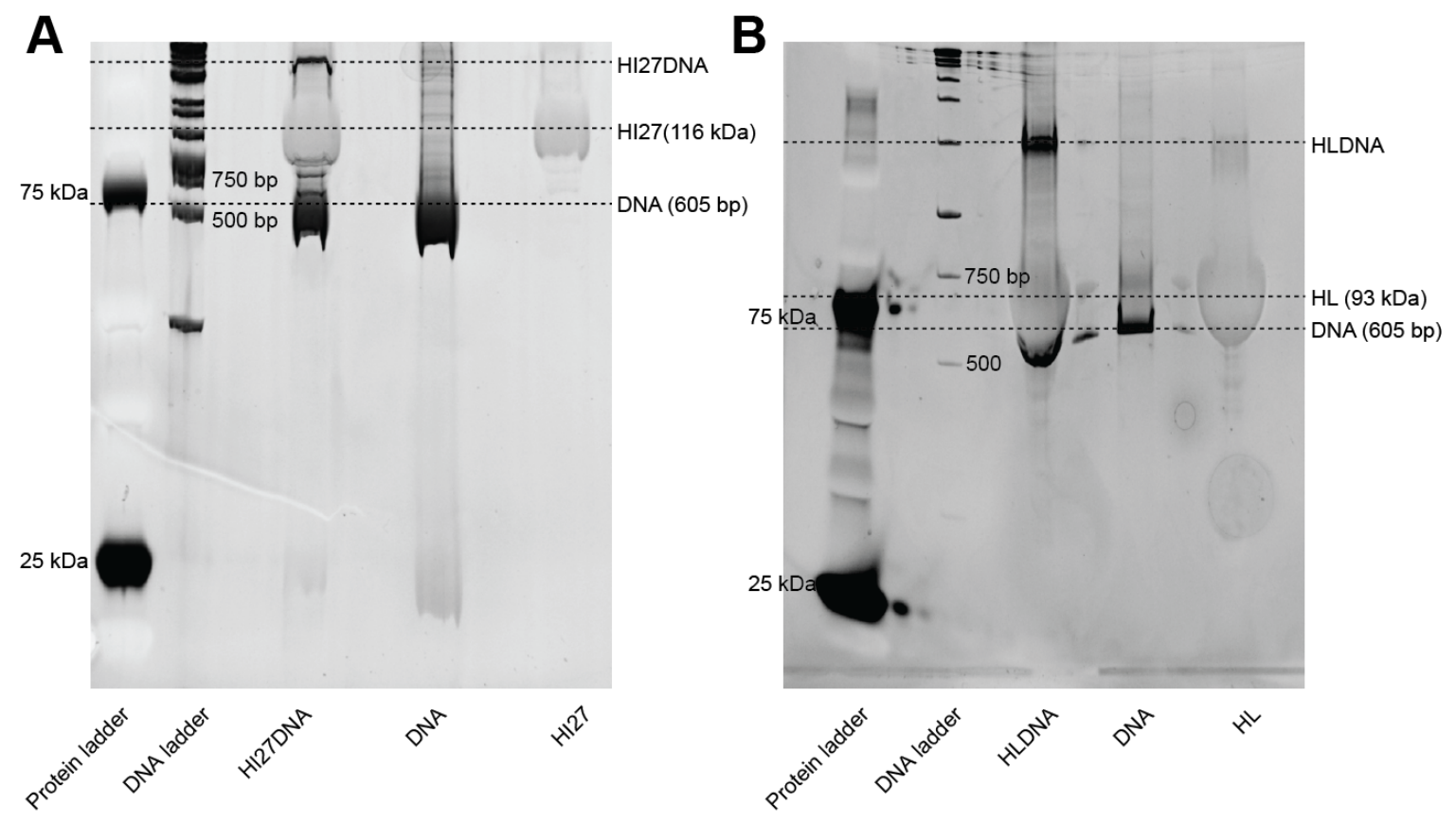

Figure S3. Staining of protein-DNA constructs with ethidium bromide, following electrophoresis on $5 \%$ to $16 \%$ polyacrylamide SDS gels. A) HaloTag-(I27 $\left.7^{47-64 C / A}\right)_{8}$ protein cross-linked with DNA. The lanes are: protein marker; DNA marker; HaloTag-(I27 $\left.7^{47-64 C / A}\right)_{8}$ DNA construct; DNA control (expected to migrate at $605 \mathrm{bp}$ ); HaloTag-(I27 $\left.{ }^{47-64 \mathrm{C} / \mathrm{A}}\right)_{8}$ control (expected to migrate at $\sim 116 \mathrm{kDa}$ ). B) HaloTag-(protein $\mathrm{L})_{8}$ protein cross-linked with DNA. The lanes are: protein marker; DNA marker; HaloTag-(protein L) $)_{8}$ DNA construct; DNA control (expected to migrate at $605 \mathrm{bp}$ ); HaloTag-(protein L) ${ }_{8}$ control (expected to migrate at $\sim 93 \mathrm{kDa}$ ). The successful reaction between HaloTag-(I2 $\left.7^{47-64 C / A}\right)_{8}$ protein and the DNA in A) and between HaloTag-(protein L) 8 protein and the same DNA segment in B) appears as a distinct band. 
FIGURE S4
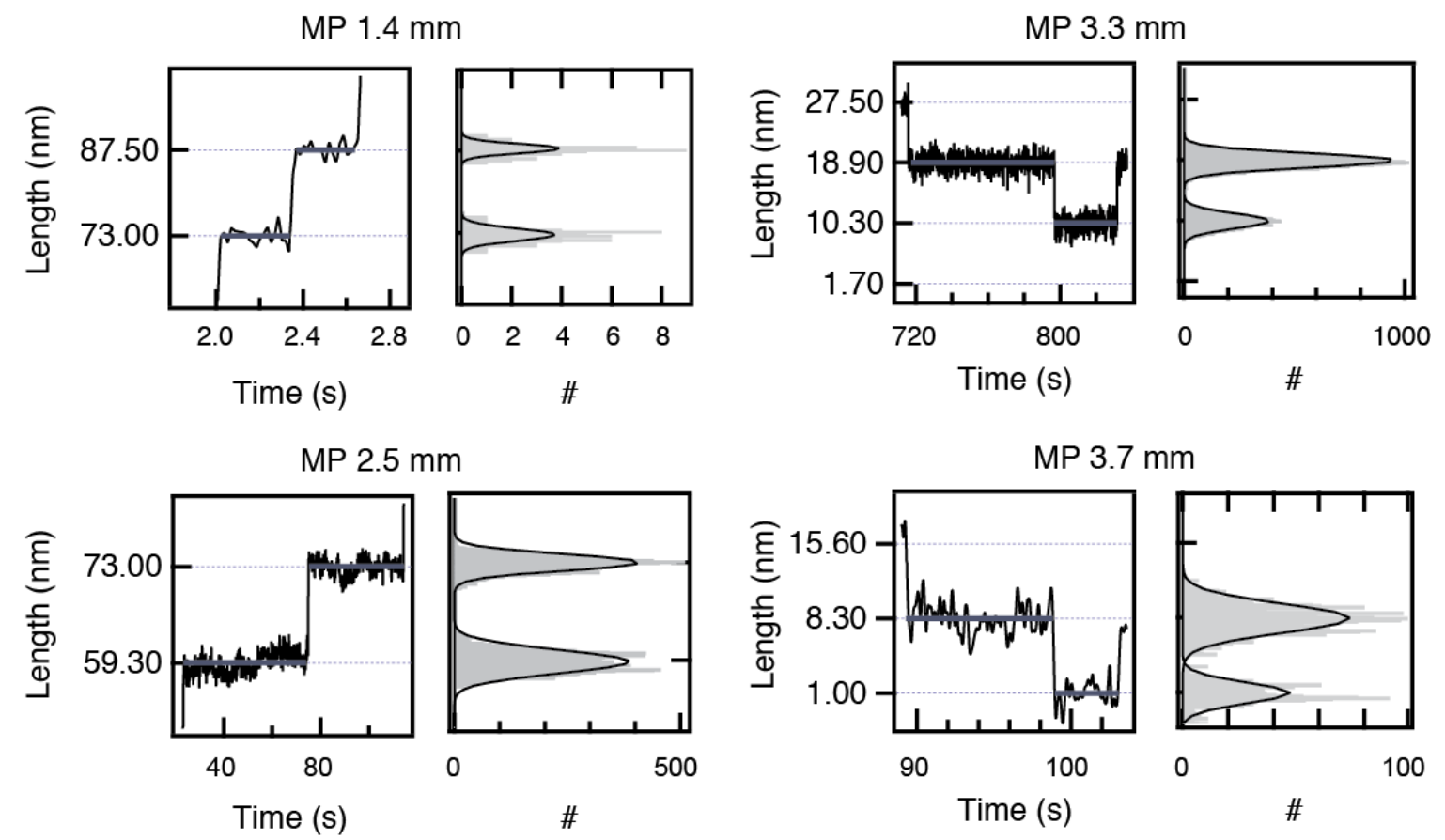

Figure S4. Step-size measurements of different magnet positions. Length histograms were obtained from the data corresponding to each step and the absolute position was measured using Gaussian fits. The size of each step was measured as the difference between the centers of the two peaks. The S.D. of was step is 1-2 $\mathrm{nm}$. The populations of the folding step sizes are normally distributed around their average. 


\section{FIGURE S5}
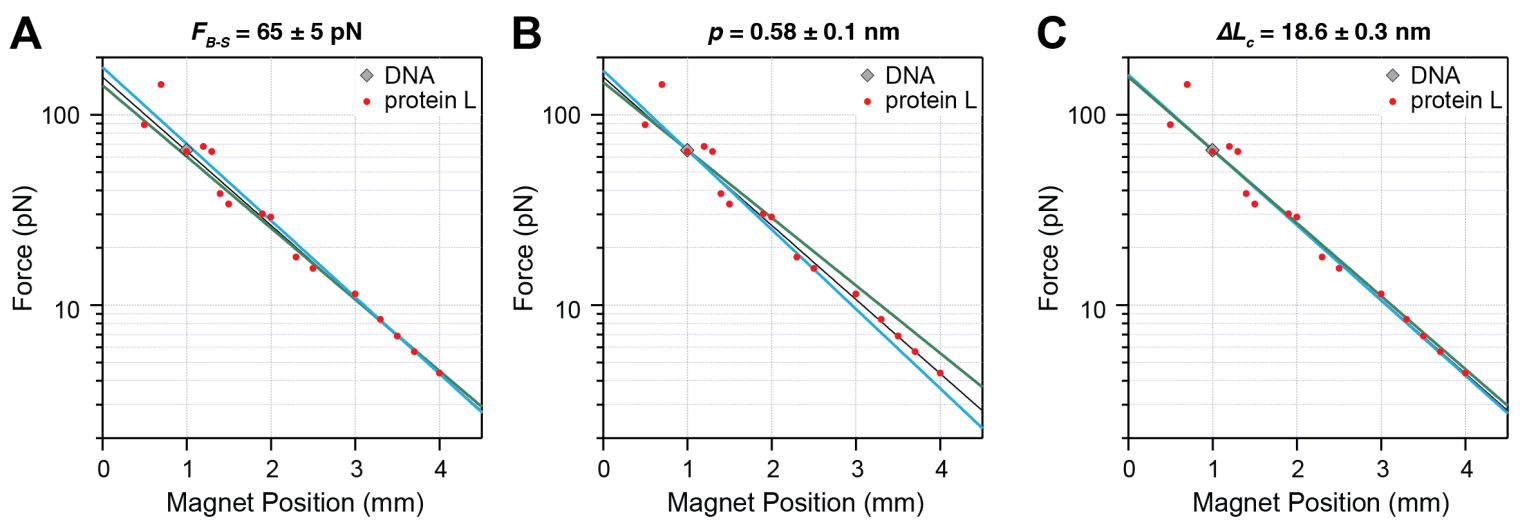

Figure S5. Testing the uncertainty of the magnet law. A) Effect of the variation of the position of the DNA B-S overstretching transition with $F_{B-S}=65 \pm 5 \mathrm{pN}$. B) Effect of the variation of the persistence length $p=0.58 \pm 0.1 \mathrm{~nm}$. C) Effect of the variation of the contour length increment $\Delta L_{c}=18.6 \pm 0.3 \mathrm{~nm}$. The points are calculated from the measured extension as a function of force (Figure 3B). The black line represents the fit using the parameters $F_{B-S}=65 \mathrm{pN}, p=0.58 \mathrm{~nm}$ and $\Delta L_{c}=18.6 \mathrm{~nm}$. The blue and green lines represent the magnet law evaluated at the upper and lower bounds of the fit parameter set as one standard deviation from the mean. The standard deviations in each of the three fit parameters were taken from previous studies. ${ }^{1 \mathrm{~b}, 4}$ 


\section{FIGURE S6}
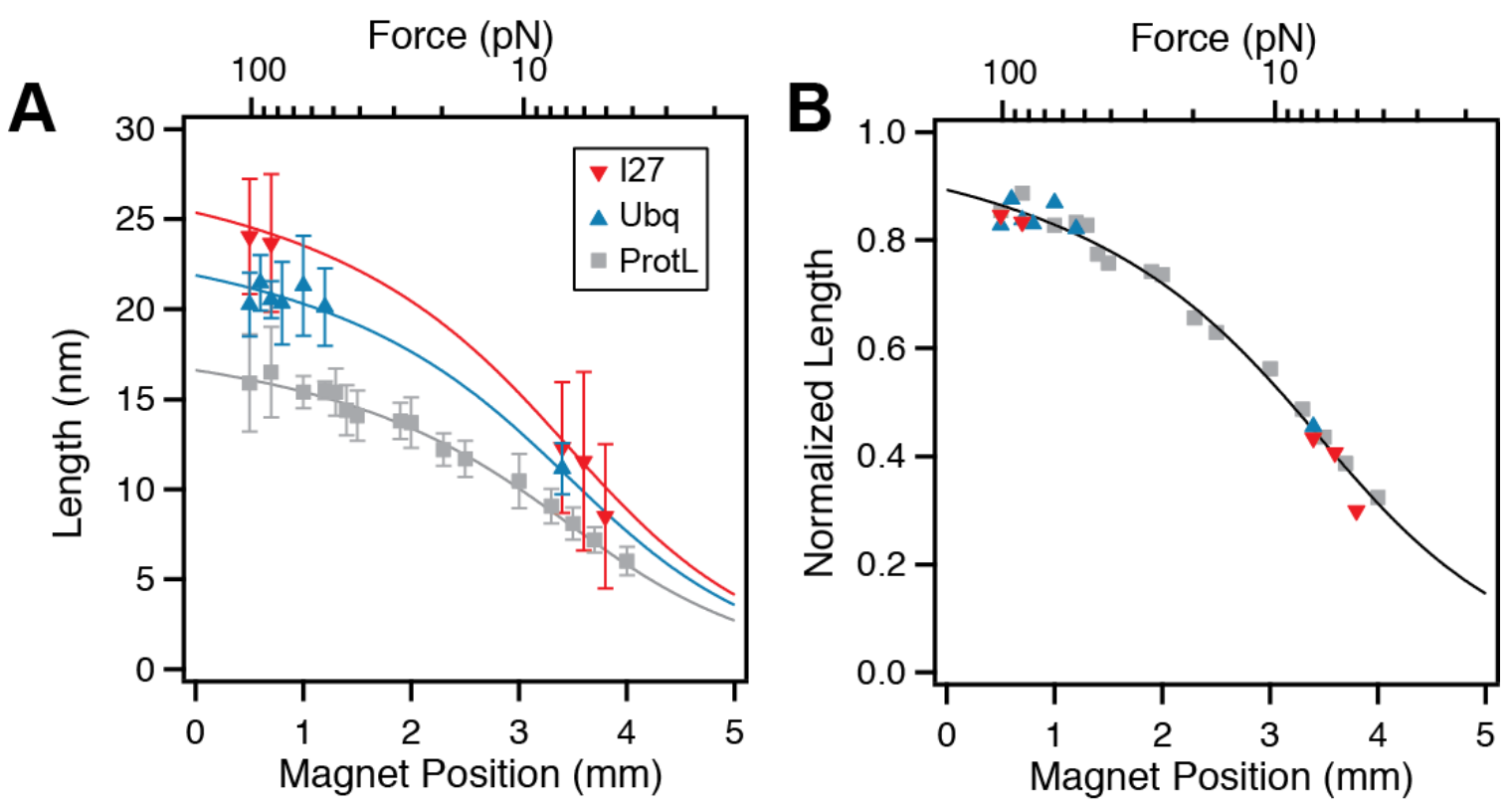

Figure S6. Length scaling of several proteins under force. A) The average step size of unfolding and folding transitions as a function of magnet position/force for four proteins: I27 from human titin, ubiquitin, and protein L. The solid lines represent the WLC law assuming a single exponential variation of force with magnet position and a contour length of $28.4 \mathrm{~nm}$ for I27, ${ }^{4}$ and of $24.5 \mathrm{~nm}$ for Ubiquitin. ${ }^{5}$ The step sizes at low force were obtained from folding transitions, while the high force points were obtained from unfolding transitions. Protein L shows folding transitions over the entire force range with smaller standard deviation $(1.1 \mathrm{~nm})$ than I27 $(3.9 \mathrm{~nm})$ and ubiquitin $(1.8 \mathrm{~nm})$, making it an ideal nano-scale ruler for calibrating the magnetic force. B) The normalized folding transitions to their contour length collapse on a single master curve. 


\section{FIGURE S7}

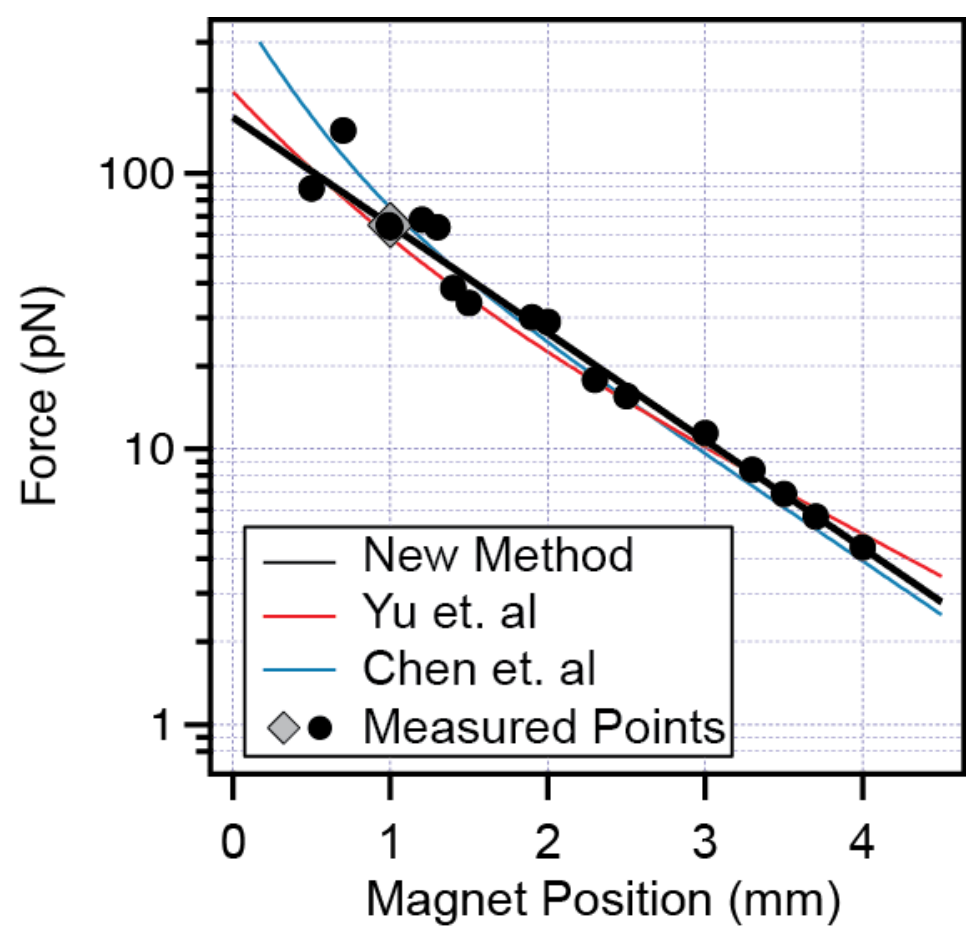

Figure S7. Comparison between the magnet law obtained with our calibration method and magnet laws obtained with fluctuation method of long DNA tethers. The points are obtained using the measured extension at different magnet positions (circles) and the B-S DNA overstretching transition (diamond). The black line represents our magnet law. The red line represents the magnet law obtained in reference ${ }^{1 \mathrm{~b}}$ for the same M-270 paramagnetic beads, and using a gap between magnets of $0.3 \mathrm{~mm}: F=-0.019+60.4 \cdot \exp (-(M P-0.4) / 0.563)+$ $56.9 \cdot \exp (-(M P-0.4) / 1.46)$. The blue line represents the magnet law obtained in reference ${ }^{6}$ for the same M-270 paramagnetic beads and the same type of magnets: $F=C \cdot(\exp (-M P / 0.36)+0.48 \cdot \exp (-M P / 1.12))$, where $C=290 \mathrm{pN}$. The figure shows good agreement between these different calibration curves at forces below $\sim 65 \mathrm{pN}$. At higher forces the calibrations tend to diverge more significantly, highlighting the difficulties in calibrating magnetic tweezers at forces higher than $65 \mathrm{pN}$. 


\section{REFERENCES}

1. (a) Huhle, A.; Klaue, D.; Brutzer, H.; Daldrop, P.; Joo, S.; Otto, O.; Keyser, U. F.; Seidel, R., Nat. Commun. 2015, 6. ; (b) Yu, Z.; Dulin, D.; Cnossen, J.; Köber, M.; van Oene, M. M.; Ordu, O.; Berghuis, B. A.; Hensgens, T.; Lipfert, J.; Dekker, N. H., Rev. Sci. Instrum. 2014, 85.

2. Vandewalle, P.; Susstrunk, S.; Vetterli, M., $\quad$ P Soc Photo-Opt Ins 2004, 5301, 374-382.

3. Velthuis, A. J. W. T.; Kerssemakers, J. W. J.; Lipfert, J.; Dekkert, N. H., Biophys. J. 2010, 99, 1292-1302.

4. $\quad$ Carrion-Vazquez, M.; Oberhauser, A. F.; Fowler, S. B.; Marszalek, P. E.; Broedel, S. E.; Clarke, J.; Fernandez, J. M., P. Natl. Acad. Sci. USA 1999, 96, 3694-3699.

5. Popa, I.; Fernandez, J. M.; Garcia-Manyes, S., J. Biol. Chem. 2011, 286, 31072-31079.

6. $\quad$ Chen, H.; Yuan, G. H.; Winardhi, R. S.; Yao, M. X.; Popa, I.; Fernandez, J. M.; Yan, J., J. Am. Chem. Soc. 2015, 137, 3540-3546. 